\title{
Effects of movement predictability on cortical motor activation
}

\author{
Paul Dassonville a,c, ScottM. Lewis ${ }^{\text {a,c }}$, Xiao-Hong Zhu b,d, Kâmil Uğurbil b,d, Seong-Gi Kim ${ }^{\text {b,d, }}$ \\ James Ashe ${ }^{\mathrm{a}, \mathrm{c}, *}$ \\ a Brain Sciences Center (11B), VAMC, 1 Veterans Drive, Minneapolis, MN 55417, USA \\ ${ }^{\mathrm{b}}$ Center for Magnetic Resonance Research, University of Minnesota Medical School, Minneapolis, MN 55455, USA \\ ${ }^{\mathrm{c}}$ Department of Neurology, University of Minnesota Medical School, Minneapolis, MN 55455, USA \\ ${ }^{\mathrm{d}}$ Department of Radiology, University of Minnesota Medical School, Minneapolis, MN 55455, USA
}

Received 20 April 1998; accepted 27 June 1998

\begin{abstract}
Humans have the ability to make motor responses to unpredictable visual stimuli, and do so as a matter of course on a daily basis. We used functional magnetic resonance imaging (fMRI) to examine the neural substrate of this behavior in six cortical motor areas. We found that five of these areas (premotor, cingulate, supplementary motor area, pre-supplementary motor area, and superior parietal lobule) showed increased activation in association with an unpredictable behavior compared to a predictable one; only the motor cortex remained unchanged. There was also a quantitative relation between the response time and functional activation in the premotor and cingulate cortex. There was less activation across all the motor areas with repetition of the motor tasks. With the exception of the pre-supplementary motor area, all areas were significantly lateralized, with a greater volume of activation in the hemisphere contralateral to the performing hand. In addition, a left hemisphere dominance was found in the activation of motor cortex and supplementary motor areas. Our results suggest that activation in motor areas is differentially and quantitatively related to higher order aspects of motor behavior such as movement predictability. (C) 1998 Elsevier Science Ireland Ltd. All rights reserved.
\end{abstract}

Keywords: fMRI; Predictability; Visually instructed movement; Sequence; Motor cortex

\section{Introduction}

In our everyday activities we regularly perform motor tasks which are over-learned, such as tying shoe laces or brushing teeth. Other motor behaviors are the result of pure volition on our part, such as reaching for a glass or picking an apple from a tree. However, in many circumstances we act on the environment in response to an unpredictable visual stimulus, for example taking corrective action when driving or catching a linedrive during a game of baseball. In the present study, we examine the activation in cortical motor areas associated with motor responses to unpredictable visual stimuli.

\footnotetext{
* Corresponding author. Tel.: + 1-612-7252282; Fax: + 1-6127252291; E-mail: ashe@maroon.tc.umn.edu
}

There has been a great deal of interest in factors thought to affect the functional activation associated with movements of the hand and arm, such as the spatial and temporal complexity (Roland et al., 1980b; Colebatch et al., 1991; Grafton et al., 1992; Matelli et al., 1993; Kawashima et al., 1994a,b; Shibasaki et al., 1993; Sadato et al., 1996), whether movements were stimulus triggered or internally generated (Roland et al., 1980a; Fox et al., 1985; Deiber et al., 1991, 1996; Rao et al., 1993, 1997; Boecker et al., 1994; Larsson et al., 1996), and the learning and acquisition of motor skills (Seitz et al., 1990, 1994; Seitz and Roland, 1992; Jenkins et al., 1994; Schlaug et al., 1994; Grafton et al., 1995; Deiber et al., 1997; Hazeltine et al., 1997; Jueptner et al., 1997a,b; Shadmehr and Holcomb, 1997). However, to our knowledge there have not been any 
functional imaging studies which have specifically addressed the issue of the predictability of a motor response. Deiber et al. (1996) have examined the functional activation associated with the preparation of movements under conditions of different amounts of information about the upcoming movement; however, the emphasis was on preparatory processes in contrast to the current study which focuses on direct motor responses to visual stimuli. Neural recordings in monkeys have been performed under conditions in which the amount of information contained in the stimulus was varied but in these cases the emphasis was on different stimulus-response mapping rules, or movement parameter specification, rather than on neural relations to response predictability (Riehle and Requin, 1989, 1995; Zhang et al., 1997).

In the present study, we contrasted two different sequences of finger movements, both of which were visually instructed in reaction time tasks. One sequence was entirely predictable while the other was randomly generated. Human subjects show changes in motor response time related to the predictability of a visual stimulus in choice response time tasks (Hyman, 1953; Hick, 1952), such as randomly generated sequences. In the current experiment we hypothesized that the behavioral changes related to predictability would correlate with changes in functional activation in cortical motor areas.

\section{Methods}

\subsection{Behavioral task}

Thirteen subjects [seven right-handed $(\mathrm{RH}$; three male, four female) and six left-handed ( $\mathrm{LH}$; four male, two female), aged 19-34 years (mean 25.5)] took part in the study. The Edinburgh inventory (Oldfield, 1971) was used to determine the handedness of each subject (laterality quotient for RH: range 33 to 100 , mean 81.2; for $\mathrm{LH}$ : range -25 to -100 , mean -62.4$)$. The effects of handedness on the pattern of functional activation have been described elsewhere (Dassonville et al., 1997). In the present investigation of the effects of movement predictability, data from LH and RH subjects have been combined, except as noted.

At the beginning of each task, the second through fifth fingers of the subject's prone semi-flexed hand rested on four low-force push buttons mounted on a small pad. The visual instruction was through the use of four annuli arranged horizontally, one corresponding to each finger, displayed on a computer screen and visible through the use of a small mirror fixed in the magnet directly above the head of the subject. Filling of an annulus instructed an extension of the corresponding digit. The annuli were filled, one at a time $(0.75$
$\mathrm{Hz}$ ), in either a randomized sequence (unpredictable task) or a regular left-to-right repeating sequence (predictable task). The subjects were informed in advance about the nature of the repeating sequence. They were instructed to move the appropriate finger as quickly as possible after the visual stimulus and fixate the visual display during both the task and the control periods. The subjects were specifically instructed not to move until the stimulus appeared.

\subsection{Experimental design}

The behavioral tasks were presented in $60 \mathrm{~s}$ blocks. The presentation of the tasks was randomized for each hand, and within each hand the order of tasks was randomized. Each task period was bracketed by two $60 \mathrm{~s}$ visual control periods (the whole comprising one $180 \mathrm{~s}$ experimental period) during which appropriate control visual stimuli (predictable or unpredictable) were shown and the subjects were instructed to attend to the stimulus but not to produce a motor response. Each 180-s experimental period was followed by a rest interval of $\approx 120 \mathrm{~s}$. There was one experimental period for each condition (hand/ task) after which the sequence of conditions was repeated; thus, subjects performed a total of eight experiments (two hand $\times$ two task $\times$ two repetitions). Each subject performed one practice session of the predictable task with the right and left hands.

\subsection{Magnetic resonance imaging}

Magnetic resonance (MR) images were obtained in a 4 Tesla whole body system equipped with an actively shielded head gradient coil insert and a quadrature head coil (SIS, Palo Alto, CA, and Siemens, Erlangen, Germany). Anatomical $T_{1}$-weighted images of the whole brain (multislice turboFLASH, echo time $(\mathrm{TE})=3 \mathrm{~ms}$, repetition time $(\mathrm{TR})=7 \mathrm{~ms}, 128 \times 128$ pixels, field of view $(\mathrm{FOV})=24 \times 24 \mathrm{~cm}^{2}, 5 \mathrm{~mm}$ slice thickness) were first obtained in coronal, sagittal and transverse planes to allow identification of the anterior and posterior commissures, and to determine the appropriate volume for the subsequent functional images. This volume was subsequently imaged in the transverse plane with $T_{1}$-weighted echo planar imaging (EPI) (four segment $\mathrm{EPI}, \mathrm{TE}=8 \mathrm{~ms}, \mathrm{TR}=42 \mathrm{~ms} /$ segment and 3 $\mathrm{s} /$ image, inversion time $=1.2 \mathrm{~s}, 128 \times 128$ pixels, FOV $=24 \times 24 \mathrm{~cm}^{2}, 5 \mathrm{~mm}$ slice thickness) to provide for accurate overlay of the functional images (Kim et al., 1995). Blood oxygen level dependent (BOLD) based functional MR images in the transverse plane $(\mathrm{TE}=25$ $\mathrm{ms}, \mathrm{TR}=50 \mathrm{~ms}, 64 \times 64$ pixels, FOV $=24 \times 24 \mathrm{~cm}^{2}$ ) were obtained with blipped EPI, with the total imaged volume extending from the superior pole of the cortex to a depth of $50 \mathrm{~mm}$ in ten slices. Functional images 
had an in-plane resolution of $3.75 \times 3.75 \mathrm{~mm}^{2}$ and a thickness of $5 \mathrm{~mm}$, and were collected every $3 \mathrm{~s}$ during each $180 \mathrm{~s}$ experiment, with 20 images collected in each of the control and task periods.

\subsection{Functional image analysis}

Functional images were first screened for movement artifact by inspecting the images and examining head motion data which were collected during image collection using a pressure sensor; those images with an excessive amount of motion were not analyzed further. The functional data were zero-filled to $128 \times 128$ pixels and then Fourier-transformed to yield a resultant nominal in-plane resolution of $1.875 \times 1.875 \mathrm{~mm}^{2}$. The data were then filtered using a Gaussian filter with FWHM of $2 \mathrm{~mm}$. Pixels with signal intensities having a coefficient of variation $>2.5 \%$ during the task control periods were masked to eliminate large vessel contributions (Kim et al., 1994). For each remaining voxel, two separate Student's $t$-tests were performed; one to test for differences between the pre-task control and the task, and the other between the post-task control and the task periods. The independent $t$-test has been shown to be both sensitive and specific for the detection of functional activation in magnetic resonance images (Xiong et al., 1996). Only those voxels that showed significant differences in both $t$-tests, each at a significance level of $P<0.05$, and were part of a group of four contiguous activated voxels were included in the analysis. The resultant significance for each voxel was $P<0.02$, calculated using a Monte Carlo simulation. Voxels activated using these criteria formed a functional activation map, which was overlaid onto the EPI anatomical images. The relatively liberal significance level used to calculate the functional maps ensured that small, but real, activations were not arbitrarily eliminated by a significance level that was too restrictive. Task effects were then determined with a repeated measures analysis of variance (ANOVA, see below) that compared these functional maps. The important issue was not so much the number of activated pixels during each condition (predictable and unpredictable) but rather the difference between the conditions. Any type I statistical errors that may have been included in the creation of the functional maps would be randomly distributed and equally likely to occur in all task conditions, and would therefore not affect the results of the ANOVA comparison between conditions.

\subsection{Statistical data analysis}

The primary datum was the number of activated voxels calculated separately for each area of interest contralateral and ipsilateral to the hand used to perform the task. Prior to statistical analysis, the number of activated voxels was transformed using a square root transformation to stabilize the variance and normalize the distribution (Snedecor and Cochran, 1989). Main effects and interactions were tested using a repeated measures analysis of variance (ANOVA). For the purposes of statistical analysis, the behavioral measures were transformed: the response time was transformed using an inverse transform, the number of errors using a square root transformation.

\subsection{Anatomical boundaries}

Regions of interest were delineated in the EPI anatomical images using anatomical landmarks in the brains of the individual subjects (Fig. 1A). Primary motor cortex was defined as the volume of cortex that included the posterior half of the precentral gyrus (including the anterior bank of the central sulcus). Premotor cortex included the anterior half of the precentral gyrus as well as the anterior bank of the precentral sulcus. Supplementary motor area (SMA) was limited to the cortex on the medial wall of the hemisphere, extending from the top of the brain to the depth of the cingulate sulcus, including the dorsal bank of the cingulate sulcus (Barbas and Pandya, 1987; Vogt and Pandya, 1987); the posterior boundary was halfway between the extension of the central and precentral sulci onto the medial surface, and the anterior boundary was defined by the vertical line drawn from the anterior commissure ((VCA), Talairach and Tournoux, 1988). Pre-supplementary motor area (PreSMA) was the extension of the SMA rostral to the VCA line to include sector D in Talairach space, from the top of the brain to the cingulate sulcus (including the dorsal bank). Cingulate motor area was contained within the cingulate gyrus inferior to SMA. Superior parietal lobule extended anteriorly to the postcentral sulcus, laterally to the intraparietal sulcus, posteriorly to the parieto-occipital sulcus, and medially to the parieto-occipital and cingulate sulci.

\section{Results}

\subsection{Behavior}

There was a significant difference (ANOVA, $P<$ $0.0001)$ in response time between tasks: unpredictable $(482.3 \pm 9.0 \mathrm{~ms}$, mean $\pm \mathrm{SEM})$, predictable $(359 \pm 7.5$ $\mathrm{ms})$. There was also a significant difference $(P<0.05)$ in response time between repetitions: repetition 1 $(431.6 \pm 11.6 \mathrm{~ms})$, repetition $2(412.4 \pm 12.8 \mathrm{~ms})$. All response times were calculated after eliminating what were regarded as anticipatory responses $(<100 \mathrm{~ms})$. Anticipatory responses occurred in $<2 \%$ of all trials, with no significant difference between the percentages 


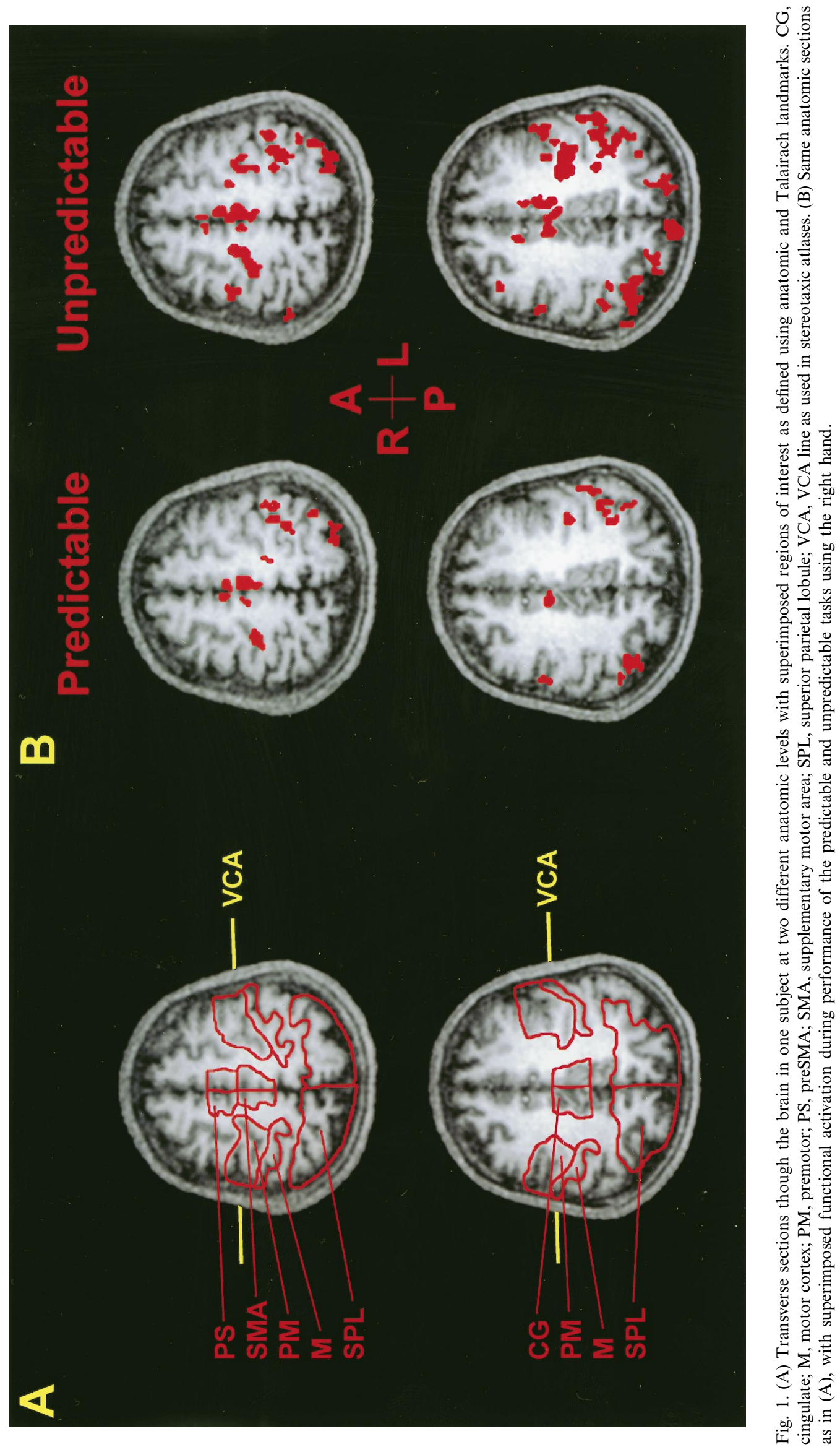


in the predictable and unpredictable tasks. The error rate was significantly different $(P<0.0005)$ between tasks: unpredictable $(0.854 \pm 0.157$ errors/block, or $2.14 \pm 0.36 \%$ of total responses), predictable $(0.326 \pm$ 0.093 , or $0.8 \pm 0.38 \%$ ). There was no main effect of repetition on the error rate.

\subsection{Task}

There was a main effect of task across all areas in the 13 subjects $(P<0.0001)$. Performance of the unpredictable task was associated with a greater volume of activation (Fig. 1(B) and Fig. 2). The total number of voxels activated in all the cortical motor areas was $709.7 \pm 56.9$ (mean \pm S.E.M.) in the unpredictable task, and $558.5 \pm 48.6$ in the predictable task. Although there was no significant task $\times$ area interaction, there are a priori reasons to expect differences in relation to task among these cortical motor areas. For this reason, we tested for task effects in the individual areas, finding significant effects $(P<0.05)$ in each area with the exception of the motor cortex (Fig. 2). The direction of the change in activation was always the same, with more activation during the unpredictable task. There was no interaction between task and hemisphere, whether hemisphere was coded as (right/left) or as (contralateral/ipsilateral).

There was a significant relation between the response time and functional activation both across all areas (Fig. 3A), and in the premotor and cingulate cortex (Fig. 3B). The focus of activation in the premotor cortex was generally within an area recognized as dorsal premotor area (Fink et al., 1997).

\subsection{Repetition}

There was a main effect of repetition across all motor areas in all subjects $(P<0.05)$ with less functional activation during the second repetition. This trend was

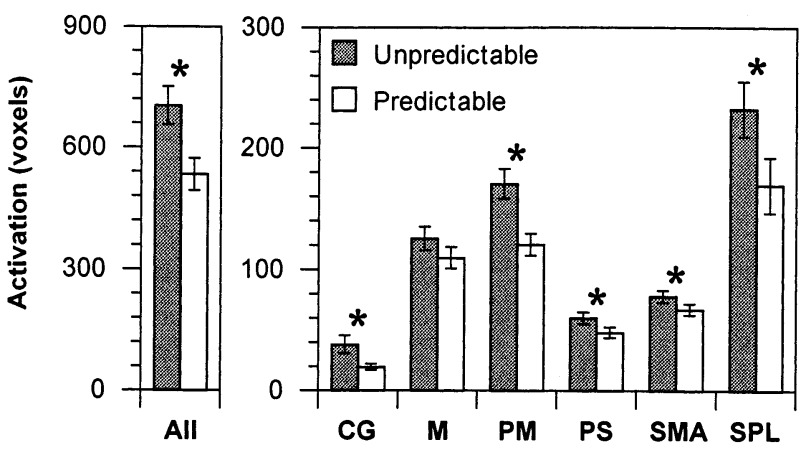

Fig. 2. Total activated volume (voxels) across all cortical motor areas (left panel) and in individual motor areas (right panel) during performance of the unpredictable (gray) and predictable (white) tasks. * Significant differences $(P<0.05)$ between tasks. Abbreviations are as in Fig. 1.
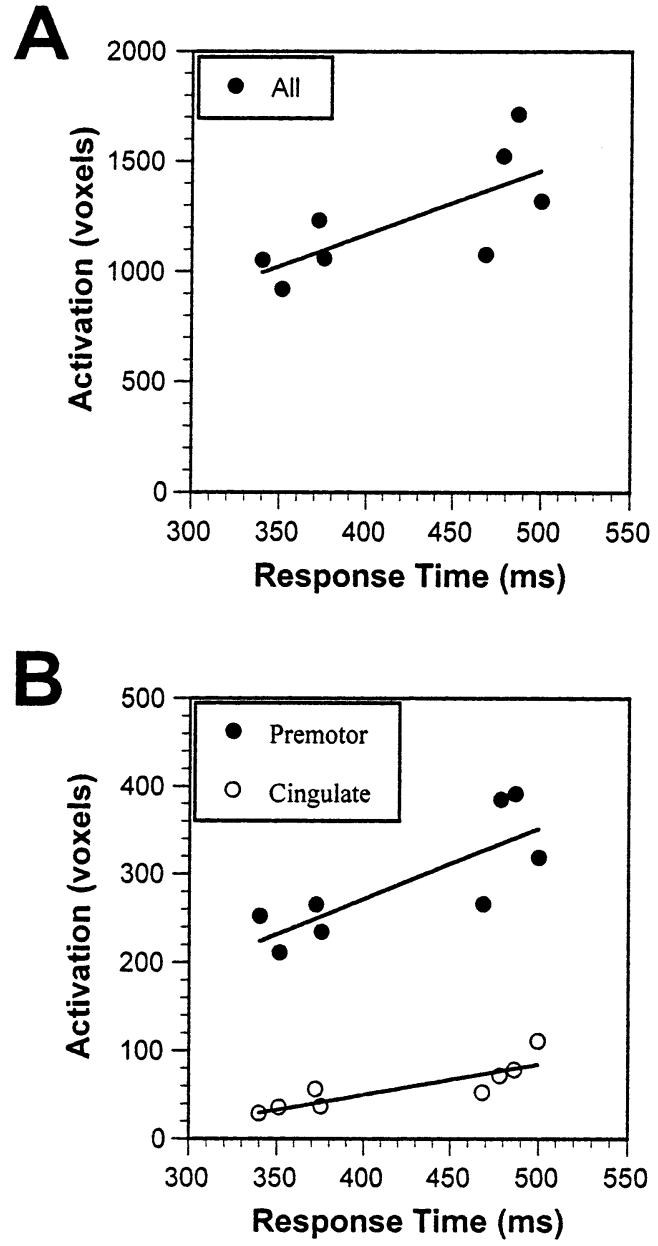

Fig. 3. (A) Functional activation across all motor areas related to the response time for each of the eight conditions [(hand (2) $\times$ task $(2) \times$ repetition (2)] in all subjects. The linear regression was significant $(P<0.05)$ with an $R^{2}$ of 0.53 . (B) Functional activation in the premotor cortex and cingulate cortex related to the response time for the eight conditions in all subjects. The linear regression was significant in the premotor cortex $(P<0.02)$ with $R^{2}$ of 0.64 , and in the cingulate cortex $(P=0.005)$ with $R^{2}$ of 0.75 .

seen in the individual cortical motor areas although it did not reach statistical significance. There was no interaction between the effects of repetition and task.

\subsection{Hemisphere (right/left)}

There was a significant main effect $(P<0.0005)$ of hemisphere in the SMA across all the subjects, with consistently greater functional activation in the left SMA than in the right. This was also true when the RH $(P<0.0001)$ and $\mathrm{LH}(P<0.0001)$ subjects were treated as independent groups. These functional differences could not be attributed to differences in the anatomic volume of the SMA, as defined by the anatomical landmarks outlined in Methods: anatomic volumes did not differ significantly (paired $t$-test) between hemispheres across subjects (right SMA, $538 \pm 179$ voxels; 


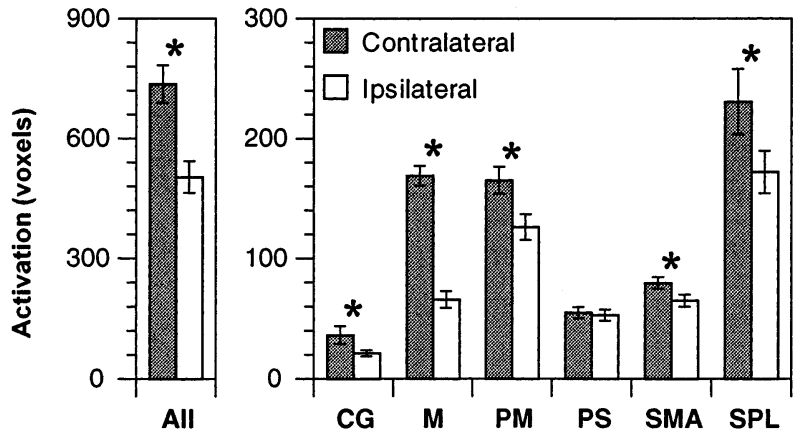

Fig. 4. Total activated volume (voxels) across all cortical motor areas (left panel) and in individual motor areas (right panel) contralateral (gray) and ipsilateral (white) to the performing hand. Functional lateralization was defined as a significant difference $(P<0.05)$ between the volume of ipsilateral and contralateral activation and is indicated with an asterisk. Abbreviations are as in Fig. 1.

left SMA, $564 \pm 134$ ) nor within RH (right SMA, $504 \pm 216$; left SMA, $492 \pm 124$ ) or LH (right SMA, $576 \pm 133$; left SMA, $648 \pm 95)$ subjects when analyzed separately. In RH subjects there was a larger volume of functional activation in the left compared to the right motor cortex $(P<0.03)$. The anatomic volume of the motor cortex in each hemisphere of the RH subjects was not significantly different (paired $t$-test: right motor cortex, $1357 \pm 345$; left motor cortex, $1430 \pm 147$ ).

\subsection{Hemisphere (contralateral/ipsilateral)}

There was a significant lateralization of activation $(P<0.0005)$ across all the motor areas, with more activation contralateral to the performing hand in all subjects (Fig. 4) and in the RH and LH subjects treated separately. There was also a significant hemisphere $x$ area interaction $(P<0.0001)$, indicating that the pattern of lateralization differed between cortical areas. Whereas preSMA showed no significant lateralization, all other areas showed a significantly greater activation in the contralateral hemisphere $(P<0.05$, Fig. 4$)$. The motor cortex had the greatest degree of lateralization, as measured by a ratio of contralateral to contralateral plus ipsilateral activation (see Table 1). There was no

Table 1

Lateralization index $[C /(C+I)]$ for each of the motor areas across conditions $^{\mathrm{a}}$

\section{$C /(C+I)$}

\begin{tabular}{ll}
\hline Pre-supplementary motor area & 0.50 \\
Supplementary motor area & 0.54 \\
Premotor & 0.56 \\
Superior parietal lobule & 0.57 \\
Cingulate & 0.63 \\
Motor cortex & 0.71
\end{tabular}

\footnotetext{
${ }^{\text {a }} C=$ number of contralateral voxels activated and $I=$ number of ipsilateral voxels.
}

interaction between task and lateralization of activation.

\section{Discussion}

The principal finding in the study was that unpredictable visually guided motor behavior was associated with an increase in activation in many cortical motor areas with the exception of the motor cortex. In the premotor and cingulate cortex this activation was quantitatively related to the behavior of the subjects as reflected by the response time to the visual stimulus. We also found that repetition of a motor behavior leads to an overall decrease in activation, which has obvious implications for the interpretation of results in imaging experiments which involve multiple repetitions of the same behavior.

\subsection{Specificity of activation in cortical motor areas}

Functional specificity within cortical motor areas, and indeed within cortical areas in general, is quite an old idea (Leyton and Sherrington, 1917). For example, Roland et al. (1980a), on the basis of one of the earlier functional imaging studies, suggested that the SMA was activated specifically during internally generated movements and particularly during movement sequences. It has since been shown that the SMA may be activated under many other different conditions, such as during instructed shoulder movements (Colebatch et al., 1991), and during simple finger movements (Fox et al., 1985). In the current study SMA was activated during both tasks, although both were externally triggered and one of the sequences was relatively simple and predictable. Data from neural studies in nonhuman primates suggest that even the most simple movements are subserved by parallel activation in many different motor areas and that it is the relative rather than the absolute activation that is important (Alexander and Crutcher, 1990a,b; Mushiake et al., 1991; Matsuzaka et al., 1992; Ashe and Georgopoulos, 1994; Halsband et al., 1994; Chen and Wise, 1995; Kalaska and Crammond, 1995; Johnson et al., 1996). The differences among motor areas therefore are more likely to be quantitative than qualitative, although subregions within areas may be functionally specific. The results of the current study, in which activation was seen in all motor areas of interest during both tasks, is consistent with a distributed network of cortical areas being responsible for motor output.

\subsection{The effect of predictability}

The unpredictability of the visual stimulus, and thus of the motor response, led to a clear increase in activa- 
tion in all of the motor areas examined with the exception of the motor cortex. The behavior in the predictable and unpredictable conditions was controlled to the extent that there were no differences in the frequency of the visual stimuli, the total number of motor responses, or in the number of movements of individual fingers. The unpredictable task was associated with changes in behavior such as an increase in response time and errors in performance. Of course, predictability was not the only factor that differed between the tasks. The attentional requirements of the subjects were also different. The differences in attention between tasks relate to the significance of, or information conveyed by, the visual stimulus. In the unpredictable task the subject relied on the visual stimulus for information about both the spatial location and the timing of the upcoming finger movement, in that the stimulus served as both a spatial instruction and a GO signal. In the predictable task the subject did not need to attend to the spatial location of the stimulus and could have used the visual stimulus merely as a GO stimulus. In fact, because the movements were paced at a constant frequency, the GO was predictable in both tasks. Different levels of attention have been shown to affect the activity of cells in motor and nonmotor areas of the frontal cortex during visuomotor tasks (Boussaoud and Wise, 1993; di Pelligrino and Wise, 1993). There were no controls for attention in the behavioral tasks since it is virtually impossible to adequately control for this factor when the tasks themselves involve different levels of predictability. By definition, the unpredictable behavior requires more attention. It is possible that during the control period the subjects mentally rehearsed the movements and it has been shown that mental rehearsal is associated with activation in many of the cortical motor areas we examined (Decety et al., 1994; Stephan et al., 1995; Roth et al., 1996). However, any activation associated with rehearsal of the movements would have been subtracted from the final images.

In addition to finding a greater volume of activation during the unpredictable task in five of the six areas examined, we also documented a quantitative relation between activation and performance. The change in activation was related to the response time in cingulate and premotor cortex. There are ample data indicating that response time increases with greater stimulus uncertainty and our data suggest that this uncertainty is an important determinant of activation in these two cortical motor areas. Although the activation was related to the response time it could also reflect other processes which may influence the response time such as motor preparation: both the premotor cortex and the cingulate motor area have been shown to be involved in preparatory processes (Picard and Strick, 1997; Wise et al., 1997). The finding that the strongest relation be- tween task and activation was seen in the premotor cortex is consistent with its prominent role in visually guided movement (see Wise et al., 1997 for review) and is also in keeping with the modulation of neural activity in this area by a whole variety of factors connecting visual stimuli to motor responses, such as angle of gaze, spatial and nonspatial visual signals and commands for motor output.

The activation in the cingulate cortex was located primarily in the caudal cingulate (CCZ in the terminology of Picard and Strick, 1996). This area has generally been shown to change activity in relation to relatively simple movements; its functional relation to motor output mirrors that of SMA (Picard and Strick, 1996). It is somewhat surprising, therefore, that we found a relation to a complex behavior (movement predictability). However, because our designation of cingulate did not include the dorsal bank of the cingulate sulcus, the activation we observed was concentrated in the ventral portion. Our results raise the possibility that caudal cingulate may actually comprise two distinct areas (dorsal and ventral) which have different relations to motor output.

\subsection{Repetition}

In the current study both the predictable and unpredictable tasks were performed once with each hand, after which the whole behavior was repeated. This gave us the opportunity to examine the effect of repetition of the same behavior over time. Repetition of the motor behavior led to a decrease in functional activation across all the motor areas. This effect was associated with a reduction in response time between repetitions, although the error rate of performance did not change. The improvement in behavioral performance, which was evident in both tasks, may have been related to a learning effect on the part of the subjects despite the fact that they had been trained in the task before the functional imaging component of the experiment. Learning in this context would be at the most general level and relate in both tasks to issues such as the appropriate pace at which to expect the stimulus presentation, the geometric arrangement of the pushbuttons, or other such task parameters unrelated to movement predictability.

The issue of the stability of functional activation associated with a particular behavior over time is an important one in functional imaging. The behaviors which are studied tend to be repeated several times both to increase the signal to noise level in the combined images, and to facilitate analysis of the activated voxels using cross correlation (Bandettini et al., 1993) or statistical parametric mapping techniques. There are few if any data on this topic in the literature, although a number of studies have suggested that the strength of 
the fMRI response in visual areas to prolonged visual stimulation may decrease over time (Frahm et al., 1996; Chen et al., 1998), at least within a subset of voxels (Chen et al., 1998). Practice in a motor task may result in a changed pattern of functional activation (Karni et al., 1995; Petersen et al., 1998). It is well known that the responses in the sensory systems may habituate over time, and may consequently be associated with a reduction in functional activation. It is possible that the effect we observed in relation to repetition would be eliminated once performance had reached a plateau. Nevertheless, it is important to remember that the time frame of our experiment was similar to that of many other experiments that use functional imaging to study motor and other systems. Therefore, we suggest that investigators should consider this issue in the design of experiments.

\subsection{Lateralization}

The control of movement is one of the clearest hemispherically-lateralized functions in the brain. Unlike other process which show functional lateralization, such as language or visual spatial function, the functional lateralization of motor control has a strong and unequivocal underlying anatomic basis (Kuypers, 1981). Approximately $90 \%$ of corticospinal fibers from each hemisphere are distributed to the contralateral spinal cord, making the control of movement predominantly a function of the contralateral hemisphere. The role of the ipsilateral corticospinal projections, particularly those emanating from the motor cortex to distal muscles, is somewhat controversial (Roland and Zilles, 1996). Neural recordings in the motor cortex of nonhuman primates (Evarts, 1966; Tanji et al., 1988) and human subjects (Goldring and Racheson, 1971) have shown that about $10 \%$ of cells relate to movements on the ipsilateral side of the body. A number of investigators have suggested that activation in ipsilateral motor cortex occurs only during movements of high spatial and temporal complexity (Kawashima et al., 1994a,b; Roland and Zilles, 1996), although this group (Kim et al., 1993a,b) and others (Kawashima et al., 1993; Chen et al., 1997) have documented the existence of ipsilateral control during more simple movement sequences. It is possible that the ipsilateral motor cortex shows more activation during complex motor tasks, yet there was no interaction between task and lateralization in the current study. Our results do not allow us to arrive at any specific conclusion as to the role of ipsilateral motor cortex activation save that it may occur during simple tasks and does not always increase with task complexity. There are data which suggest that ipsilateral activation in the motor cortex may be related to the extent of handedness (Dassonville et al., 1997).
Nonprimary motor areas are thought to be less functionally lateralized than the motor cortex, and there is evidence from recordings in nonhuman primates to support this view (Tanji and Kurata, 1981; Tanji et al., 1988). Data from the current work and other studies on human subjects also support this point (Weiller et al., 1992; Kawashima et al., 1993; Kim et al., 1993a,b). The reason for the less obvious lateralization in nonprimary motor areas is presumably related to the fact that these areas are less directly involved in motor output, have no monosynaptic motoneuronal connections, and are involved with more general aspects of motor function such as visual guidance (premotor cortex), timing and internal generation (SMA), and higher somatosensory function (superior parietal lobule).

\subsection{Hemisphere dominance for movement}

Only one previous study in human subjects has specifically addressed the issue of hemisphere dominance in the control of movement (Kim et al., 1993b). The authors concluded that the left motor cortex was the dominant one as it was activated during movements of both the contralateral and ipsilateral hand. This finding is consistent with data from human subjects who may exhibit bilateral symptoms after stroke involving the left hemisphere (Haaland et al., 1987). In the current study we found that there was a larger volume of activation in the left motor cortex compared to the right, despite the fact that the anatomic volume of motor cortex did not differ between hemispheres across subjects. We should point out that we compared the total anatomic volume of the motor cortex in each hemisphere rather then the portion presumably related to arm movement as has been done in other studies (Amunts et al., 1996; White et al., 1997). The increased activation in the left motor cortex reached significance only in RH subjects; again this is consistent with previous work in which the dominance of the left motor cortex was most evident in RH subjects (Kim et al., 1993b).

We also found that the left SMA was dominant during the performance of the tasks across subjects. It is not clear whether this is: (i) the result of a general relation between SMA activation and motor output, as is the case for the motor cortex, (ii) related to the fact that the left SMA is more active during movement sequences predictable or unpredictable, or (iii) indeed further evidence that the left hemisphere is the dominant one for the control of movement. The issue of hemispheric dominance of human SMA during movement has not been addressed specifically in the literature; in part, this may be due to technical factors as it has been difficult to reliably assign SMA activity to one or the other hemisphere because of inadequate spatial resolution. 


\section{Acknowledgements}

We thank J. Bullis for help with data processing, and A.P. Georgopoulos, A. Verbanov and G. Oehlert for helpful discussion. This work was supported by the National Institute of Health grant NS 32437, the Charles A. Dana Foundation, the Department of Veterans Affairs, and the American Legion Chair in Brain Sciences and P41 RR09079, a National Research Resource (NIH) Grant.

\section{References}

Alexander, G.E., Crutcher, M.D., 1990a. Preparation for movement: neural representations of intended direction in three motor areas of the monkey. J. Neurophysiol. 64, 133-149.

Alexander, G.E., Crutcher, M.D., 1990b. Neural representations of the target (goal) of visually guided arm movements in three motor areas of the monkey. J. Neurophysiol. 64, 164-177.

Amunts, K., Schlaug, G., Schleicher, A., Steinmetz, H., Dabringhaus, A., Roland, P.E., Zilles, K., 1996. Asymmetry in the human motor cortex and handedness. Neuroimage 4, 216-222.

Ashe, J., Georgopoulos, A.P., 1994. Movement parameters and neuronal activity in motor cortex and area 5. Cereb. Cortex 6, 590-600.

Bandettini, P.A., Jesmanowicz, A., Wong, E.C., Hyde, J.S., 1993. Processing strategies for time course data sets in functional MRI of the human brain. Magn. Reson. Med. 30, 161-173.

Barbas, H., Pandya, D.N., 1987. Architecture and frontal cortical connections of the premotor cortex (area 6) in the rhesus monkey. J. Comp. Neurol. 256, 211-228.

Boecker, H., Kleinschmidt, A., Requardt, M., Hanicke, W., Merboldt, K.D., Frahm, J., 1994. Functional cooperativity of human cortical motor areas during self-paced simple finger movements: a high-resolution MRI study. Brain 117, 1231-1239.

Boussaoud, D., Wise, S.P., 1993. Primate frontal cortex: neuronal activity following attentional versus intentional cues. Exp. Brain Res. 95, 15-27.

Chen, L.L., Wise, S.P., 1995. Supplementary eye field contrasted with the frontal eye field during acquisition of conditional oculomotor associations. J. Neurophysiol. 73, 1122-1134.

Chen, R., Gerloff, C., Hallett, M., Cohen, L.G., 1997. Involvement of the ipsilateral motor cortex in finger movements of different complexities. Ann. Neurol. 41, 247-254.

Chen, W., Zhu, X.-H., Kato, T., Andersen, P., Ugurbil, K., 1998. Spatial and temporal differentiation of fMRI BOLD response in primary visual cortex of human brain during sustained visual stimulation. Magn. Reson. Med., 39, 520-527.

Colebatch, J.G., Deiber, M.P., Passingham, R.E., Friston, K.J., Frackowiak, S.J., 1991. Regional cerebral blood flow during voluntary arm and hand movements in human subjects. J. Neurophysiol. 65, 1392-1402.

Dassonville, P., Zhu, X.-H., Ugurbil, K., Kim, S.-G., Ashe, J., 1997. Functional activation in motor cortex reflects the direction and the degree of handedness. Proc. Natl. Acad. Sci. USA 94, 1401514018.

Decety, J., Perani, D., Jeannerod, M., et al., 1994. Mapping motor representations with positron emission tomography. Nature 371, $600-602$.

Deiber, M.-P., Passingham, R.E., Colebatch, J.G., Friston, K.J., Nixon, P.D., Frackowiak, R.S.J., 1991. Cortical areas and the selection of movement: a study with positron emission topography. Exp. Brain Res. 84, 393-402.
Deiber, M.-P., Ibanez, V., Sadato, N., Hallet, M., 1996. Cerebral structures participating in motor preparation in humans: a positron emission tomography study. J. Neurophysiol. 75, 233247.

Deiber, M.-P., Wise, S.P., Honda, M., Catalan, M.J., Grafman, J., Hallett, M., 1997. Frontal and parietal networks for conditional motor-learning - a positron emission tomography study. J. Neurophysiol. 78, 977-991.

di Pelligrino, G., Wise, S.P., 1993. Effects of attention on visuomotor activity in the premotor and prefrontal cortex of a primate. Somatosen. Mot. Res. 10, 245-262.

Evarts, E.V., 1966. Pyramidal tract neuron activity associated with a conditional hand movement in the monkey. J. Neurophysiol. 29, 1011-1027.

Fink, G.R., Frackowiak, R.S.J., Pietrzyk, U., Passingham, R.E., 1997. Multiple nonprimary motor areas in the human cortex. J. Neurophysiol. 77, 2164-2174.

Fox, P.T., Fox, J.M., Raichle, M.E., Burde, R.M., 1985. The role of cerebral cortex in the generation of voluntary saccades: a positron emission tomographic study. J. Neurophysiol. 54, 348-369.

Frahm, J., Kruger, G., Merboldt, K.D., Kleinschmidt, A., 1996. Dynamic uncoupling and recoupling of perfusion and oxidative metabolism during focal brainactivation in man. Magn. Reson. Med. 35, 143-148.

Goldring, S., Racheson, R., 1971. Human motor cortex: sensory input data from single neuron recordings. Science 175, 14931495.

Grafton, S.T., Mazziotta, J.C., Presty, S., Friston, K.J., Frackowiak, R.S., Phelps, M.E., 1992. Functional anatomy of human procedural learning determined with regional cerebral blood flow and PET. J. Neurosci. 12, 2542-2548.

Grafton, S.T., Hazeltine, E., Ivry, R., 1995. Functional mapping of sequence learning in normal humans. J. Cog. Neurosci. 7, 497510 .

Haaland, K.Y., Harrington, D.L., Yeo, R., 1987. The effects of task complexity on motor performance in left and right CVA patients. Neuropsychologia 25, 783-794.

Halsband, U., Matsuzaka, Y., Tanji, J., 1994. Neuronal activity in the primate supplementary, pre-supplementary and premotor cortex during externally and internally instructed sequential movements. Neurosci. Res. 20, 149-155.

Hazeltine, E., Grafton, S.T., Ivry, R., 1997. Attention and stimulus characteristics determine the locus of motor-sequence encoding. A PET study. Brain 120, 123-140.

Hick, W.F., 1952. On the rate of gain of information. Q. J. Exp. Psychol. 4, 11-26.

Hyman, R., 1953. Stimulus information as a determinant of reaction time. J. Exp. Psychol. 45, 188-196.

Jenkins, I.H., Brooks, D.J., Nixon, P.D., Frackowiak, R.S.J., Passingham, R.E., 1994. Motor sequence learning: a study with positron emission tomography. J. Neurosci. 14, 3775-3790.

Johnson, P.B., Ferraina, S., Bianchi, L., Caminiti, R., 1996. Cortical networks for visual reaching: physiological and anatomical organization of frontal and parietal lobe arm regions. Cereb. Cortex 6, $102-119$.

Jueptner, M., Stephan, K.M., Frith, C.D., Brooks, D.J., Frackowiak, R.S.J., Passingham, R.E., 1997a. Anatomy of motor learning. I. Frontal cortex and attention to action. J. Neurophysiol. 77, $1313-1324$.

Jueptner, M., Frith, C.D., Brooks, D.J., Frackowiak, R.S.J., Passingham, R.E., 1997b. Anatomy of motor learning. II. Subcortical structures and learning by trial and error. J. Neurophysiol. 77, 1325-1337.

Kalaska, J.F., Crammond, D.J., 1995. Deciding not to GO: neuronal correlates of response selection in a $\mathrm{GO} / \mathrm{NOGO}$ task in primate premotor and parietal cortex. Cereb. Cortex 5, 410-428. 
Karni, A., Meyer, G., Jezzard, P., Adams, M.M., Turner, R., Ungerleider, L.G., 1995. Functional MRI evidence for adult motor cortex plasticity during motor skill learning. Nature 377, 155-159.

Kawashima, R., Yamada, K., Kinomura, S., Yamaguchi, T., Matsui, H., Yoshioka, S., 1993. Regional cerebral blood flow changes of cortical motor areas and prefrontal areas in humans related to ipsilateral and contralateral hand movement. Brain Res. 623, $33-40$.

Kawashima, R., Roland, P.E., O'Sullivan, B.T., 1994a. Activity in the human primary motor cortex related to ipsilateral hand movements. Brain Res. 663, 251-256.

Kawashima, R., Roland, P.E., O'Sullivan, B.T., 1994b. Fields in human motor areas involved in preparation for reaching, actual reaching, and visuomotor learning: a positron emission tomography study. J. Neurosci. 14, 3462-3474.

Kim, S.-G., Ashe, J., Georgopoulos, A.P., Merkle, H., Ellermann, J.M., Menon, R.M., Ogawa, S., Ugurbil, K., 1993a. Functional imaging of the human motor cortex at high magnetic field. J. Neurophysiol. 69, 297-302.

Kim, S.-G., Ashe, J., Hendrich, K., Ellermann, J.M., Merkle, H., Ugurbil, K., Georgopoulos, A.P., 1993b. Motor cortex, hemispheric asymmetry, and handedness. Science 261, 615-617.

Kim, S.-G., Hendrich, K., Hu, X., Merkle, H., Ugurbil, K., 1994. Potential pitfalls of functional mri using conventional gradient-recalled echo techniques. NMR Biomed. 7, 69-74.

Kim, S.-G., Hu, X., Adriany, G., Ugurbil, K., 1995. Fast interleaved echo-planar imaging with navigator: high resolution anatomic and functional images at 4 Tesla. Magn. Reson. Med. 35, 895-902.

Kuypers, H.G.J.M., 1981. Anatomy of the descending pathways. Handbook of Physiology. The Nervous System I. American Physiological Society, Bethesda, MD, pp. 597-666.

Larsson, J., Gulyas, B., Roland, P.E., 1996. Cortical representation of self-paced finger movement. Neuroreport 7, 463-468.

Leyton, A.S.F., Sherrington, C.S., 1917. Observations on the excitable cortex of the chimpanzee, orang-utan, and gorilla. Q. J. Exp. Physiol. 11, 135-222.

Matelli, M., Rizzolatti, G., Bettinardi, V., Gilardi, M.C., Perani, D., Rizzo, G., Fazio, F., 1993. Activation of precentral and mesial motor areas during the execution of elementary proximal and distal arm movements: a PET study. Neuroreport 4, 1295-1298.

Matsuzaka, Y., Aizawa, H., Tanji, J., 1992. A motor area rostral to the supplementary motor area (presupplementary motor area) in the monkey: neuronal activity during a learned motor task. J. Neurophysiol. 68, 653-662.

Mushiake, H., Inase, M., Tanji, J., 1991. Neuronal activity in the primate premotor, supplementary and precentral motor cortex during visually guided and internally determined sequential movements. J. Neurophysiol. 66, 705-718.

Oldfield, R.C., 1971. The assessment and analysis of handedness: The Edinburgh inventory. Neuropsychologia 9, 97-113.

Petersen, S.E., van Mier, H., Fiez, J.A., Raichle, M.E., 1998. The effects of practice on the functional anatomy of task performance. Proc. Natl. Acad. Sci. USA 95, 853-860.

Picard, N., Strick, P.L., 1996. Motor areas of the medial wall: a review of their location and functional activation. Cereb. Cortex 6, 342-353.

Picard, N., Strick, P.L., 1997. Activation on the medial wall during remembered sequences of reaching movements in monkeys. J. Neurophysiol. 77, 2197-2201.

Rao, S.M., Binder, J.R., Bandettini, P.A., Hammeke, T.A., Yetkin, F.Z., Jesmanowitz, A., List, L.M., 1993. Functional magnetic resonance imaging of complex human movements. Neurology 43, 2311-2318.

Rao, S.M., Deborah, H.L., Haaland, K.Y., Bobholz, J.A., Cox, R.W., Binder, J.R., 1997. Distributed neural systems underlying the timing of movements. J. Neurosci. 17, 5528-5535.

Riehle, A., Requin, J., 1989. Monkey primary motor and premotor cortex: single-cell activity related to prior information about direction and extent of an intended movement. J. Neurophysiol. 61, $534-549$.

Riehle, A., Requin, J., 1995. Neuronal correlates of the specification of movement direction and force in four cortical areas of the monkey. Behav. Brain Res. 70, 1-13.

Roland, P.E., Larsen, B., Lassen, N.A., Skinhoj, E., 1980a. Supplementary motor area and other cortical areas in organization of voluntary movements in man. J. Neurophysiol. 43, 118-136.

Roland, P.E., Skinhoj, E., Lassen, N.A., Larsen, B., 1980b. Different cortical areas in man in organization of voluntary movements in extrapersonal space. J. Neurophysiol. 43, 137-150.

Roland, P.E., Zilles, K., 1996. Functions and structures of the motor cortices in humans. Curr. Opin. Neurobiol. 6, 773-781.

Roth, R., Decety, J., Raybaudi, M., et al., 1996. Possible involvement of primary motor cortex in mentally simulated movement-a functional magnetic resonance imaging study. Neuroreport 7, 1280-1284.

Sadato, N., Campbell, G., Ibanez, V., Deiber, M.P., Hallet, M., 1996. Complexity affects regional cerebral blood flow change during sequential finger movements. J. Neurosci. 16, 2693-2700.

Schlaug, G., Knorr, U., Seitz, R.J., 1994. Inter-subject variability of cerebral activations in acquiring a motor skill — a study with positron emission tomography. Exp. Brain Res. 98, 523-534.

Seitz, R.J., Roland, P.E., 1992. Learning of sequential finger movements in man: a combined kinematic and positron emission tomography (PET) study. Eur. J. Neurosci. 4, 154-165.

Seitz, R.J., Roland, P.E., Bohm, C., Greitz, T., Stone-Elander, S., 1990. Motor learning in man: a positron emission tomographic study. Neuroreport 1, 57-60.

Seitz, R.J., Canavan, A.G., Yaguez, L., et al., 1994. Successive roles of the cerebellum and premotor cortices in trajectorial learning. Neuroreport 5, 2541-2544.

Shadmehr, R., Holcomb, H., 1997. Neural correlates of motor memory consolidation. Science 277, 821-825.

Shibasaki, H., Sadato, N., Lyshkow, H., et al., 1993. Both primary motor cortex and supplementary motor area play an important role in complex finger movement. Brain 116, 1387-1398.

Snedecor, G.W., Cochran, W.G., 1989. Statistical Methods. Iowa State University Press, Ames, IA.

Stephan, K.M., Fink, G.R., Passingham, R.E., Silbersweig, D., Ceballos-Baumann, A.O., Frith, C.D., Frackowiak, R.S., 1995. Functional anatomy of the mental representation of upper extremity movements in healthy subjects. J. Neurophysiol. 73, 373-386.

Talairach, J., Tournoux, P., 1988. Co-planar Stereotaxic Atlas of the Human Brain. Thieme, New York

Tanji, J., Kurata, K., 1981. Contrasting neuronal activity in supplementary and precentral motor cortex of monkeys I. Brain Res. 222, $155-158$.

Tanji, J., Okano, K., Sato, K.C., 1988. Neuronal activit incortical motor areas related to ipsilateral, contralateral and bilateral digit movements of the monkey. J. Neurophysiol. 60, 325-343.

Vogt, B.A., Pandya, D.N., 1987. Cingulate cortex of the rhesus monkey: II. Cortical afferents. J. Comp. Neurol. 262, 271-289.

Weiller, C., Chollet, F., Friston, K.J., Wise, R.J., Frackowiak, R.S., 1992. Functional reorganization of the brain in recovery from striatocapsular infarction in man. Ann. Neurol. 31, 463-472.

White, L.E., Andrews, T.J., Hulette, C., Richards, A., Groelle, M., Paydarfar, J., Purves, D., 1997. Structure of the human sensorimotor system 2 lateral symmetry. Cereb. Cortex 7, 31-47.

Wise, S.P., Boussaoud, D., Johnson, P.B., Caminiti, R., 1997. Premotor and parietal cortex-corticocortical connectivity and combinatorial computations. Ann. Rev. Neurosci. 20, 25-42.

Xiong, J., Gao, J.-H., Lancaster, J.L., Fox, P.T., 1996. Assessment and optimization of functional MRI analyses. Hum. Brain Mapp. 4, $153-167$.

Zhang, J., Reihle, A., Requin, J., Kornblum, S., 1997. Dynamics of single neuronal activity in monkey primary motor cortex related to sensorimotor transformation. J. Neurosci. 17, 2227-2246. 